\title{
Fiber optic humidity sensor based on silk fibroin interference films
}

\author{
Zbigniew Opilski*1 ${ }^{*}$, Marcin Procek ${ }^{1,2}$, Salvador D. Aznar-Cervantes ${ }^{3}$, Jose Luis Cenis ${ }^{3}$, Xavier Muñoz Barbel ${ }^{4}$ \\ ${ }^{1}$ Department of Optoelectronics, Silesian University of Technology, Krzywoustego 2, 44-100 Gliwice, Poland, \\ ${ }^{2}$ Department of Electronics Design, Mid Sweden University, Sundsvall, 85170, Sweden, \\ ${ }^{3}$ Inst. Murciano de Investigación y Desarrollo Agrario y Alimentario (IMIDA), La Alberca (Murcia), 30150, Spain, \\ ${ }^{4}$ Instituto de Microelectrónica de Barcelona (IMB-CNM, CSIC), Bellaterra (Barcelona), 08193, Spain
}

Received May 14, 2020; accepted June 24, 2020; published June 30, 2020

\begin{abstract}
The article presents an inexpensive and simple method of fabrication of fiber optic interference relative humidity $(\mathrm{RH})$ sensors based on silk fibroin (SF) films. The sensors were made on standard multimode telecommunications optical fibers using a dip-coating method and examined by using broadband light sources. A measuring stand was presented, at which the basic sensor parameters were measured, as well as measured parameters.
\end{abstract}

Relative humidity (RH) sensing is important for many fields of application such as indoor and outdoor air monitoring, automotive industry, medical, agriculture, chemical areas and many others [1]. Silk fibroin (SF) produced by Bombyx Mori silkworms is a biopolymer with many desirable properties like mechanical strength, biocompatibility, biodegradability, thermal stability, ease of processing, being also implantable and edible [2]. Its processing takes place in an aqueous environment at room temperature (RT) and neutral $\mathrm{pH}$. The above is recommended as a modern material whose technology is sustainable and ecological [3].

In the literature, several papers show the possibility of application of SF to $\mathrm{RH}$ sensing in optical, $\mathrm{THz}$ and capacitance type sensors $[4 \div 7]$. However, so far fiber optic (FO) humidity sensor based on SF have not been reported. FO sensors have a number of advantages like high environmental resistance, resistance to electrical noises, small dimensions, high sensitivity, wide dynamic range, low cost and others [8]. Relatively high refractive index (1.54), film-forming properties and humidity dependent dimensions and optical properties make SF a good candidate for RH sensing [5].

This paper presents an SF based interferometric FO sensor for room temperature (RT) humidity sensing in a wide concentration range.

FO sensor heads were prepared using a dip-coating method at RT, where an aqueous solution of silk fibroin with a concentration of about $3 \% \mathrm{w} / \mathrm{v}$ was used. Sensors were manufactured at the end of standard multimode FO. During all subsequent operations, the fiber remained connected to the measuring system described in the next

\footnotetext{
*E-mail: zbigniew.opilski@ polsl.pl
}

part of the paper. This made it possible to follow the spectrum of light reflected from the fiber tip continuously. The fiber was prepared for cutting and cut in a standard manner. Immediately after cutting, the fiber tip was immersed for several seconds in an SF solution. After removal, the fiber was placed vertically for several seconds, with the tip pointing up to dry. During this operation, the water evaporated to the extent that the interference spectrum of the light reflected from the sensor layer stabilized. To fix the resulting SF layer, the fiber tip was immersed in methyl alcohol for $1 \mathrm{~min}$, and taken out to dry. Alcohol treatment removes the remaining water from the SF layer and leads to its crosslinking.

Before crosslinking, the layer can be washed with water, while after that, because of $\beta$-sheet molecular formation, the film begins to be water resistant and another layer can be applied increasing its thickness. This process can be repeated, but it involves the risk of deteriorating the surface quality.

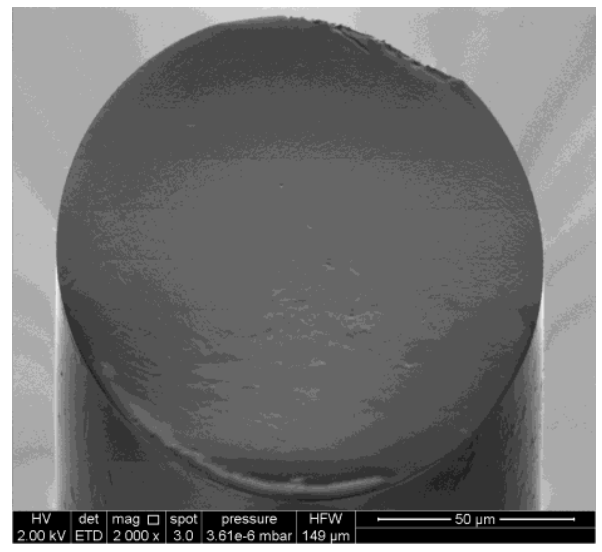

Fig. 1. Scanning electron microscope image of the front of a sensing head (SF film on FO tip).

It can be seen in Fig. 1 from the scanning electron microscope (SEM, FEI, Inspect S50) that the thickness of the layer directly at the edges is higher than in the middle. However, in the area where the core is located, the layer is much more uniform. Our previous paper [6] shows that SF is creating, on $\mathrm{SiO}_{2} / \mathrm{Si}$ substrates, uniform films with small roughness $(\mathrm{RMS}=0.67 \pm 0.01 \mathrm{~nm} @ 5 \mu \mathrm{m} \times 5 \mu \mathrm{m}$ measured 
by atomic force microscopy) when a spin coating method is used. Some minor irregularities like micro-craters and micro-hills are to be observed in the films obtained by a deep-coating method presented here. However, a relatively high interference signal, exceeded $30 \%$ despite the low contrast of the refractive index between the FO core and the SF layer, shows that the film is uniform in the area of the fiber core. What is more, for multiple tested structures the interference signal was very similar, which shows that the fabrication process is repeatable.

The humidity sensing properties of prepared SF films were tested in the system, as shown in Fig. 2. The sensor layer system made at the end of the optical fiber was placed in a measuring chamber with a volume of about $30 \mathrm{~mm}^{3}$. During measurements, carried out at a $\mathrm{RT}=24 \pm 1^{\circ} \mathrm{C}$, a constant stream of air of $500 \mathrm{ml} / \mathrm{min}$ was flowing through the chamber. The RH level of air flowing through the chamber was controlled by two mass flow controllers (MFCs) connected to dry synthetic air and a bubbler humidifier, respectively. Dry air and humid air were mixed in proper proportions before the measurement chamber. The system includes a relative humidity sensor type SHT75 (Sensirion), which measured temperature and relative humidity every 1 second as a RH reference.

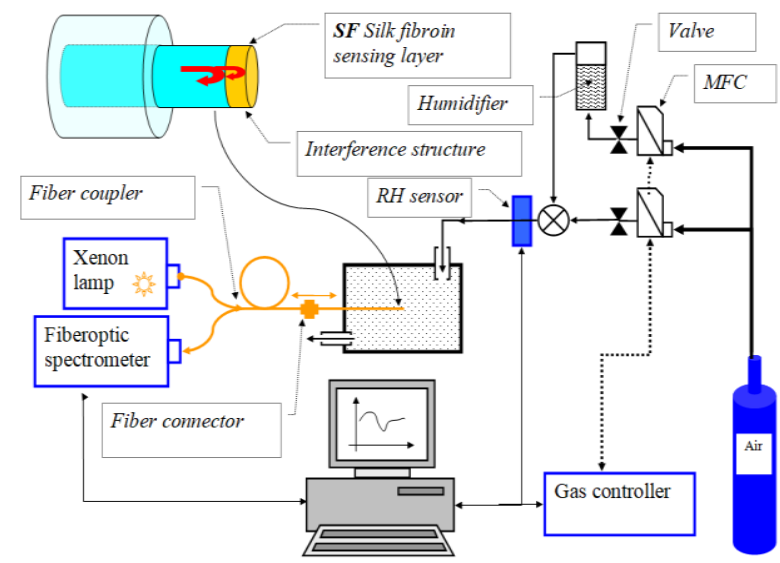

Fig. 2. Measuring system (MFC-Mass Flow Controller).

The FO with the tested sensor head was connected to a common Y-type splitter arm. The first of the available splitter ports was connected to a broadband light source a xenon lamp $(75 \mathrm{~W})$. The second to the spectrometer HR2000 + ES (Ocean Optics), measuring in the range from $200 \mathrm{~nm}$ to $1100 \mathrm{~nm}$. The SF layer on the FO tip operates as an interference film. Interferences occur between the light reflected from the interface between the core face and the SF layer and the interface between the SF layer and air. The reflection coefficients from these interfaces are so high that the interference contrast reaches up to $30 \%$. The interference system uses multi-mode fiber and white, non-polarized light. The choice of standard multimode FO is justified by its desirable simplicity and

http://www.photonics.pl/PLP cost efficiency of the system. Such FO can be easily coupled with a broadband light source. The higher area of the core is also crucial because it increases an area of the $\mathrm{SF} /$ core interface making it less sensitive to possible morphology irregularities. The lack of light polarization in the system with multimode FO does not distort considerably the phenomenon of light interference, and on the other hand, greatly simplifies the measurement system, which reduces its price.

Using a fiber optic spectrometer, the spectrum of light reflected from the layered interference structure was recorded. In the paper previously mentioned [6] we showed that as a result of water molecules absorption by the SF layer, its thickness increases in a reversible manner (at the same time the refractive index decreases slightly). The thickness growth is much stronger than the refractive index drop, and as a result, the optical path in the layer increases with an RH level increment in the full investigated range. This shifts the interference spectrum recorded by the interferometer towards red. The system recorded spectra once per 1 second. Examples of spectra recorded for different humidity levels are presented in Fig. 3.

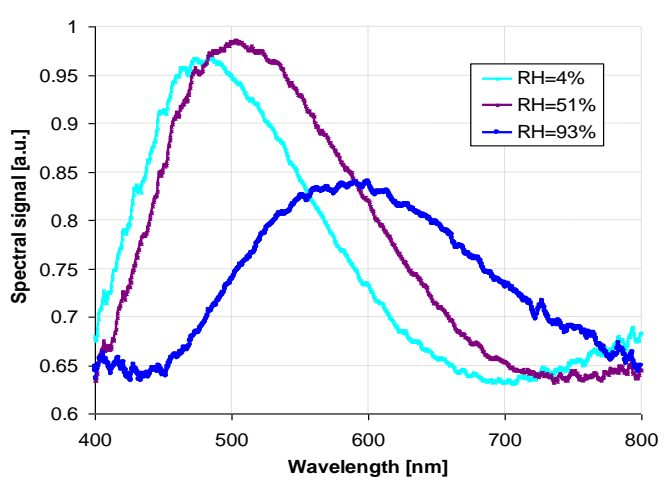

Fig. 3. Spectral characteristics of light reflected from the sensor layer recorded at three different relative humidities.

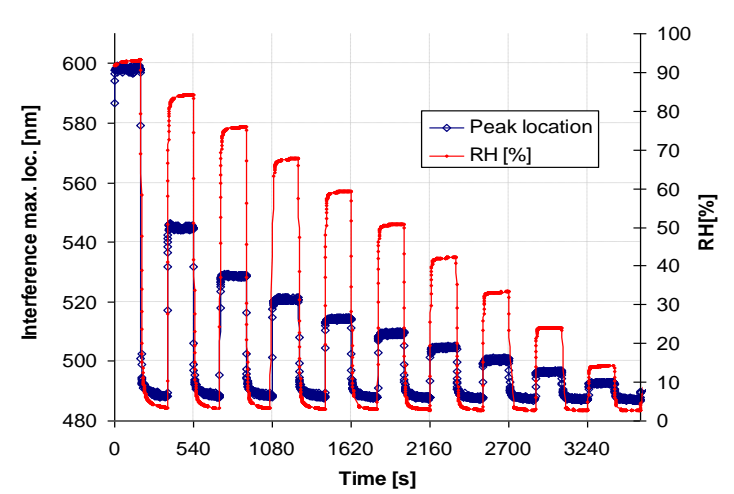

Fig. 4. Change in the location of the maximum interference caused by changes in relative humidity as a function of time.

Figure 4 presents both recorded changes in the position of interference maximum caused by changes in RH and the $\mathrm{RH}$ itself (monitored by reference sensor) as a function of 
time. The change in the location of interference maximum is significant because, as a result of a $90 \%$ relative humidity change, the interference maximum shifts by about $110 \mathrm{~nm}$. However, the changes are not linear over the entire range of humidity changes, but only monotonic. Figure 5 shows the relationship between the position of interference maximum and relative humidity.

Visible are two areas whose slope is significantly different. The first area from $\mathrm{RH}=4 \%$ to just over $\mathrm{RH}=70 \%$, where we observe rather linear changes and the second - for the largest RH, where the dependence is rather exponential.

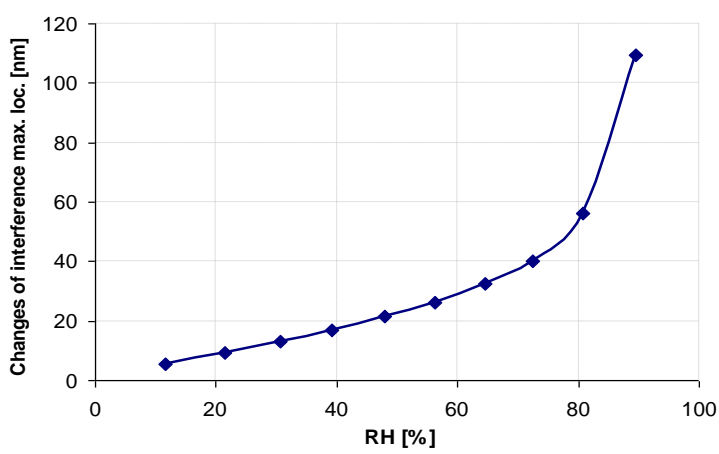

Fig. 5. The relationship between the location of the maximum interference and changes in relative humidity.

Estimates made on the basis of optical path measurement show that the thickness of the sensor layer, in relative humidity $\mathrm{RH}=4 \%$ is $\mathrm{d}=251 \pm 2 \mathrm{~nm}$, while at humidity $\mathrm{RH}=93 \%$ this thickness increases to $\mathrm{d}=313 \pm 2 \mathrm{~nm}$. Thus, layer thickness increases by about $25 \%$ in the measured $\mathrm{RH}$ range. This changes in the film thickness are caused by the $\mathrm{H}_{2} \mathrm{O}$ molecules interaction with weak hydrogen bonds between SF chains, which leads to an increase in the structure dimensions [4]. As we observe the increase in the structure sensitivity for higher RH, it seems that the interchain spaces in SF become wider and as a result, they become even more susceptible to penetration by subsequent water molecules. Also, the polarity of $\mathrm{H}_{2} \mathrm{O}$ molecules may play a role in attracting other polar molecules. The decrease of interference contrast, which is visible in Fig. 3 for $\mathrm{RH}=93 \%$, clearly shows that homogeneity and surface conditions of the SF film deteriorate at a high $\mathrm{RH}$ content. What is more, the refraction index of SF decreases with humidity growth [6], which decreases the reflection in the SF/FO interface.

Figure 4, but most of all Fig. 6, shows that the sensor response time is relatively short. Even shorter than that of the commercial sensor used as a reference. In addition, this time decreases with increasing humidity, which can be clearly observed in Fig. 6, where response and regeneration times are summarized. This trend also shows that water molecules can more easily get out from the volume of the SF film if the interchain distances are higher because of higher RH.
The sensor response time varies from about $6 \mathrm{~s}$ to about $3 \mathrm{~s}$. The regeneration time is greater and as humidity increases, it decreases from approx. $22 \mathrm{~s}$ to approx. $3 \mathrm{~s}$. This dynamic parameter shows that the proposed sensor is fast enough for most potential applications.

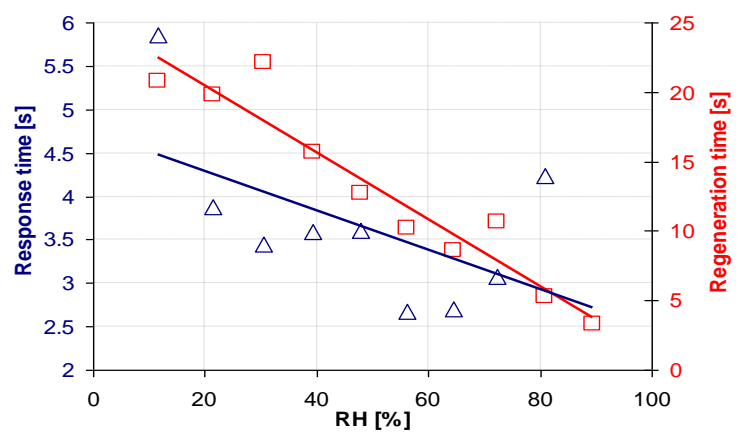

Fig.6. Sensor response and regeneration time vs. relative humidity.

In summary, one can express the opinion that silk fibroin is a promising sensor material for measuring humidity in a wide range of concentrations in RT conditions. The sensor has been fully made of non-toxic and biocompatible materials. The sensor technology presented is ecological repeatable, simple and inexpensive. Since the presented tests were carried out at RT and the interfering gasses effect is not investigated here, in further research one should examine the impact of temperature on sensor sensitivity to $\mathrm{RH}$ and cross selectivity with other gases (especially common polar agents like $\mathrm{NH}_{3}$ and alcohols vapors).

As in this paper we presented a laboratory idea of measurement which requires a spectrometer, in the future work we will focus on cheaper methods like amplitude monitoring for single or mutual light sources like LEDs or lasers.

The present work was partially sponsored by the Silesian University of Technology, Faculty of Electrical Engineering within the grants BKM/571/RE4/2019 and 260/BK/RE4/2019.

\section{References}

[1] Y.-G. Han, Sensors 19(23), 5196 (2019).

[2] L.D. Koh, Y. Cheng, C.P. Teng, Y.W. Khin, X.J. Loh, S.Y. Tee et al., Prog. Polim. Sci. 46, 86 (2015).

[3] H. Tao, D.L. Kaplan, F.G. Omenetto, Adv. Mater. 24, 2824 (2012).

[4] Q. Li, N. Qi, Y. Peng, Y. Zhang, L. Shi, X. Zhang et al., RSC Adv. 7(29), 17889 (2017).

[5] S.K. Hwan, H.C. Sung, B. Roy, S. Kim, Y.H. Ahn, Opt. Expr. 26(26), 33575 (2018).

[6] M. Procek, Z. Opilski, A.M. Maqueda, X. M. Berbel, S.D. AznarCervantes, J.L. Cenis, C.D. Horna, , Proc. SPIE 11204, 0277-786X, (2019).

[7] Y. Luo, Y. Pei, X. Feng, B. Lu, L. Wang, Mater. Lett. 260, 126945 (2020).

[8] E. Maciak, Sensors 19(3), 629 (2019). 